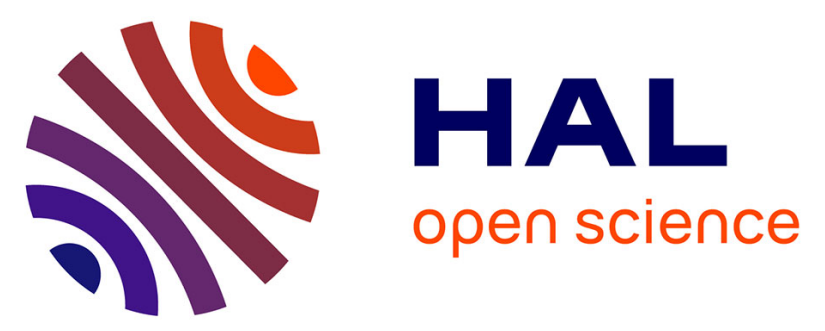

\title{
Un exemple de sédimentation carbonatée holocène de type travertineux sur le piémont nord du Haut Atlas (Maroc) :1a coupe de Makhfamane I
}

André Weisrock, Georgette Delibrias, Jean-Claude Miskovsky, A. Dutour, J P Adolphe

\section{To cite this version:}

André Weisrock, Georgette Delibrias, Jean-Claude Miskovsky, A. Dutour, J P Adolphe. Un exemple de sédimentation carbonatée holocène de type travertineux sur le piémont nord du Haut Atlas (Maroc) :1a coupe de Makhfamane I. Méditerranée: revue géographique des pays méditerranéens, 1986, 57 (1), pp.39 - 44. 10.3406/medit.1986.2366 . hal-03541312

\author{
HAL Id: hal-03541312 \\ https://hal.science/hal-03541312
}

Submitted on 24 Jan 2022

HAL is a multi-disciplinary open access archive for the deposit and dissemination of scientific research documents, whether they are published or not. The documents may come from teaching and research institutions in France or abroad, or from public or private research centers.
L'archive ouverte pluridisciplinaire HAL, est destinée au dépôt et à la diffusion de documents scientifiques de niveau recherche, publiés ou non, émanant des établissements d'enseignement et de recherche français ou étrangers, des laboratoires publics ou privés. 


\title{
Un exemple de sédimentation carbonatée holocène de type
} travertineux sur le piémont nord du Haut Atlas (Maroc) :1a coupe de Makhfamane I

\author{
André Weisrock, Georgette Delibrias, Jean-Claude Miskovsky, A. Dutour, J P Adolphe
}

\section{Abstract}

The detritic Holocène filling of the Makhfamane dale show a silty carbonated fluvial bank deposited during wet environment (travertine-type deposit), between 8000 and $7000 \mathrm{y}$. BP. Earlier was the sedimentation of eolian, than torrential types. This show that more humid conditions occur in the South-Western Morocco after the dry Lower Holocene period.

\section{Résumé}

Le comblement détritique holocène du vallon de Makhfamane montre une passée limoneuse carbonatée qui s'est déposée entre 8000 et 7000 ans BP, en régime aquatique calme (dépôt de type travertineux), alors que la sédimentation antérieure est éolienne, puis torrentielle. L'humidification du climat après la période sèche de I 'Holocène inférieur au SW du Maroc est ainsi confirmée.

\section{Citer ce document / Cite this document :}

Weisrock André, Delibrias Georgette, Miskovsky Jean-Claude, Dutour A., Adolphe J P. Un exemple de sédimentation carbonatée holocène de type travertineux sur le piémont nord du Haut Atlas (Maroc) :1a coupe de Makhfamane I. In: Méditerranée, troisième série, tome 57, 1-2-1986. Travertins LS et évolution des paysages holocènes dons le domaine méditerranéen. pp. 39-44;

doi : https://doi.org/10.3406/medit.1986.2366

https://www.persee.fr/doc/medit_0025-8296_1986_num_57_1_2366

Fichier pdf généré le 25/08/2021 


\section{LES TRAVERTINS HOLOCENES D'AFRIQUE DU NORD, D'ITALIE ET D'ESPAGNE}

\author{
Un exemple de sédimentation carbonatée \\ holocène de type travertineux sur le piémont \\ nord du haut atlas (Maroc) : la coupe de \\ Makhfamane I.
}

$$
1
$$

\author{
A. WEISROCK* \\ G. DELIBRIAS** \\ J.C. MISKOVSKI* \\ A. DUTOUR* \\ J.P. ADOLPHE*
}

Résumé - Le comblement détritique holocène du vallon de Makhfamane montre une passée limoneuse carbonatée qui s'est déposée entre 8000 et 7000 ans BP, en régime aquatique calme (dépót de type travertineux), alors que la sédimentation antérieure est éolienne, puis torrentielle. L'humidification du climat après la période sèche de l'Holocène inférieur au $S W$ du Maroc est ainsi confirmée.

Abstract - The detritic Holocene filling of the Makhfamane dale show a silty carbonated fluvial bank deposited during wet environment (travertine-type deposit), between 8000 and 7000 y. BP. Earlier was the sedimentation of eolian, than torrential types. This show that more humid conditions occur in the South-Western Morocco after the dry Lower Holocene period.

Au Sud-Ouest de Marrakech, le piémont nord du Haut Atlas est constitué par de très vastes cônes de déjection (fig.1). La vallée de l'oued N'fis entaille, au sortir de la montagne, un grand cône ancien très complexe, essentiellement tributaire du bassin-versant de l'oued Amizmiz. Cette énorme accumulation détritique date à coup sûr du Quaternaire ancien puisque tout le système des terrasses alluviales de l'oued N'fis, soit 5 à 6 terrasses étagées, s'y trouve emborté. La surface sommitale du grand cône est protégée par une carapace calcaire très karstifiée et de plus en plus amenuisée par les labours. Aussi estelle souvent réduite à des promontoires ou même à de hautes buttes isolées par une multitude de ravins rayonnants tributaires du N'fis ou même du Tensift. L'exploration des coupes de ces vallons montre qu'il y existe au minimum deux périodes de dépôts carbonatés au Pléistocène supérieur et à l'Holocène.

La première, constituée de calcaires lacustres et palustres en bancs subhorizontaux, a été placée jusqu'ici dans le Quaternaire moyen en raison d'arguments morphologiques (A. Dutour, 1985) car ces dépôts couronnent les restes de terrasses dominant les fonds de talweg d'une trentaine de mètres (coupes d'Agadir Issouel, Taouililt et oued R'bib). Deux datations C14 sur coquilles prélevées au sein de ces niveaux (GIF 6583 et GIF 6795) ont fourni des âges respectifs de $25000 \pm 900$ ans B.P. (oued R'bib) et $26400 \pm 700$ ans B.P. (Taouililt). Cette phase de sédimentation carbonatée précédant un ravinement important serait donc plutôt du Pléistocène supérieur, dans l'é tat actuel des connaissances.

En revanche, la seconde, qui correspond à une partie du remblaiement de fond de vallée postérieur au grand ravinement précédent, est indubitablement holocène. Elle est étudiée ici à partir de la coupe du Chabet el Khemis, près du douar Makhfamane.

\footnotetext{
* U.A.722 CNRS Paléoclimats et paléoenvironnements en régions arides.

* Centre des Faibles Radioactivités CNRS-CEA.
} 


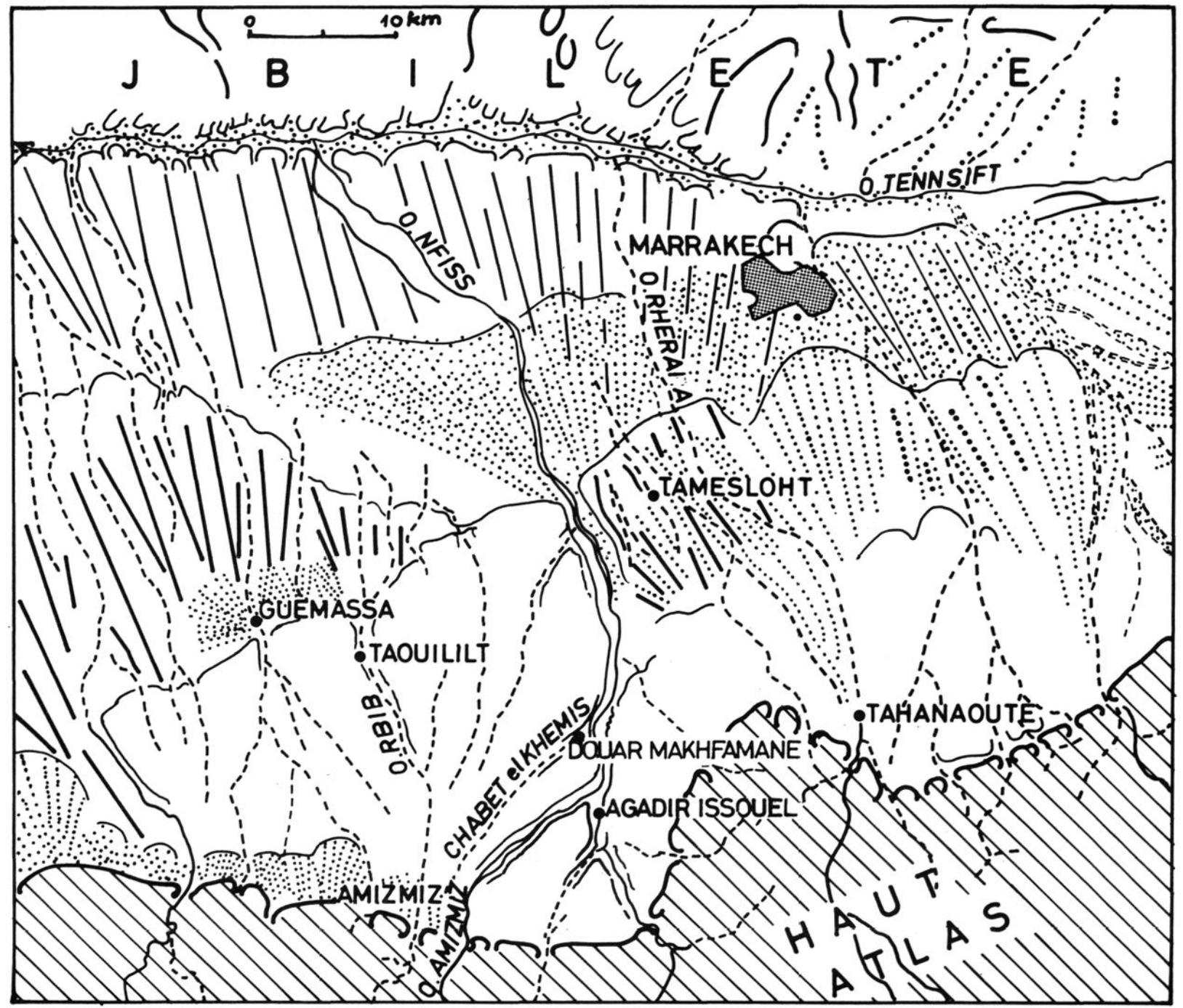

Fig. 1 : LE PIEMONT NORD DU HAUT ATLAS AU SUD.OUEST DE MARRAKECH, CARTE DE LOCALISATION (d'après A. Andre et G. Beaudet, 1970, simplifie).

La coupe MAKHFAMANE I se situe à $1 \mathrm{~km}$ à l'Est du village du même nom, à l'aval du vallon dit Chabet el Khemis, non loin du barrage sur l'oued N'fis. C'est une coupe naturelle due à l'écoulement actuel du vallon, qui entaille le remblaiement holocène sur $3 \mathrm{~m}$ d'épaisseur.

Ce remblaiement montre une alternance de dépôts fluviatiles plus ou moins grossiers et de dépôts de type palustre ou travertineux de faciès plus fin et nettement calcaire. On peut y distinguer 3 unités majeures : (fig. 2)

- l'unité de base I essentiellement sableuse et caillouteuse, visible sur 1 mètre;

- l'unité principale II, épaisse d'un peu plus d'1,50 m, formée d'un dépôt limoneux blanc-gris à texture prismatique, entrecoupé par 3 lentilles sableuses à coquilles (Helix, Melanopsis, Bivalves dulcicoles) et à débris de charbons de bois. Un lit continu de Bivalves dulcicoles se trouve à peu près en son milieu;

- l'unité supérieure III, à nouveau très grossière, vient fossiliser sans ravinement notable une ébauche de sol brun-clair qui s'est développé au sommet de l'unité II.

\section{I - ANALYSE SEDIMENTOLOGIQUE DES UNITES I ET II}

\section{Granulométries}

Le sédiment travertineux principal (subdivisions 10 et 12) est assez fin (61 à $71 \%<a ̀$ mm, cf. fig. 2) et plus limoneux que sableux par rapport aux autres dépôts (tab. 1). 
MAKHFAMANE I

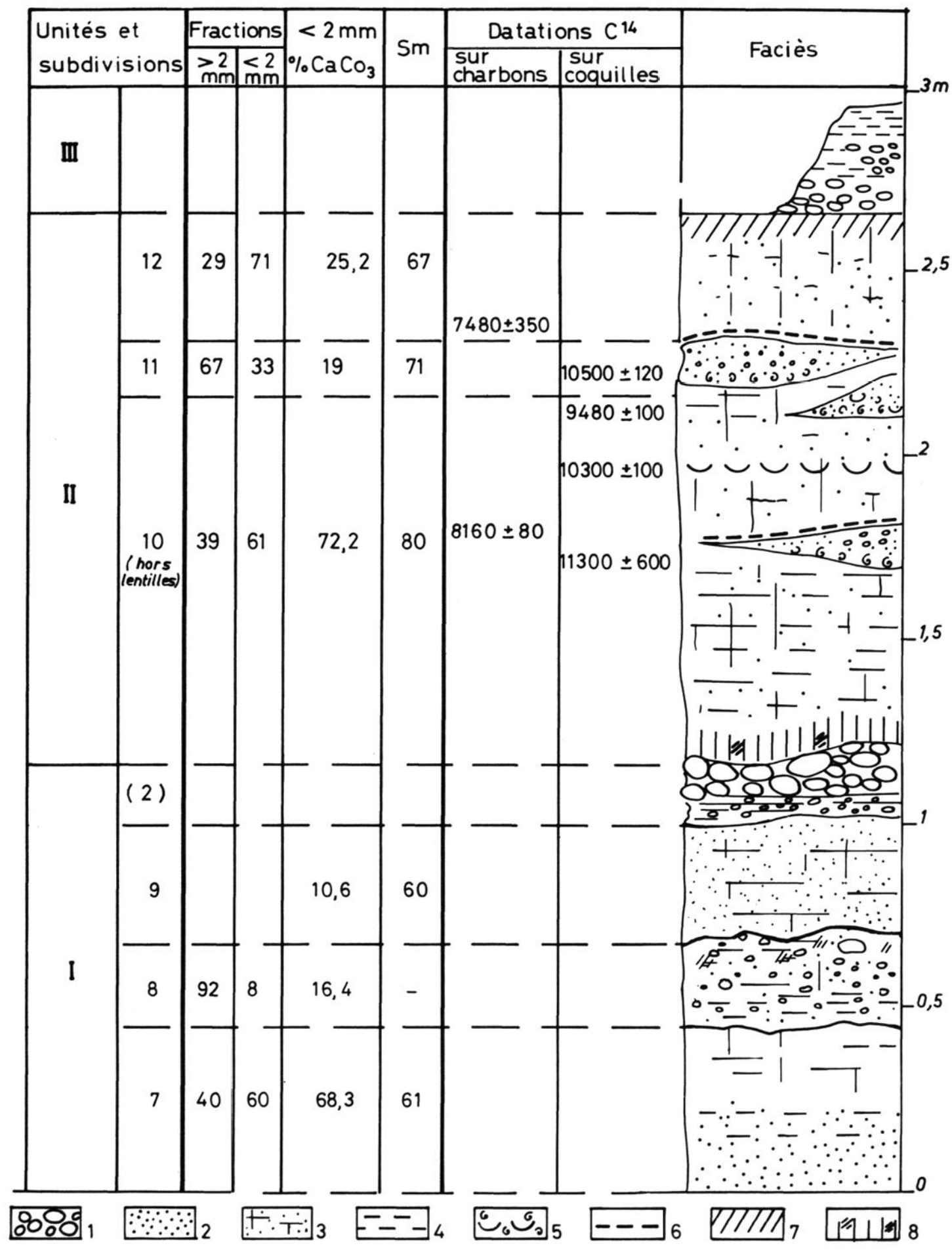

Fig. 2 : LA COUPE DE MAKHFAMANE I

1. Galets ; 2. Sables ; 3. Limons calcaires ; 4. Argiles ; 5 . Coquilles : 6. Charbons de bois ; 7. Brunification : 8. Hydromorphis et ferruginisations. 


\begin{tabular}{|c|c|c|c|c|c|c|}
\hline Echantillons & 70,0 & 80,0 & 90,0 & 10,0 & 11,0 & 12,0 \\
\hline$\%$ & 72,6 & 79,0 & 43,0 & 30,8 & 70,0 & 20,0 \\
\hline
\end{tabular}

Tab. 1 : POURCENTAGES SABLES/LIMONS + ARGILES

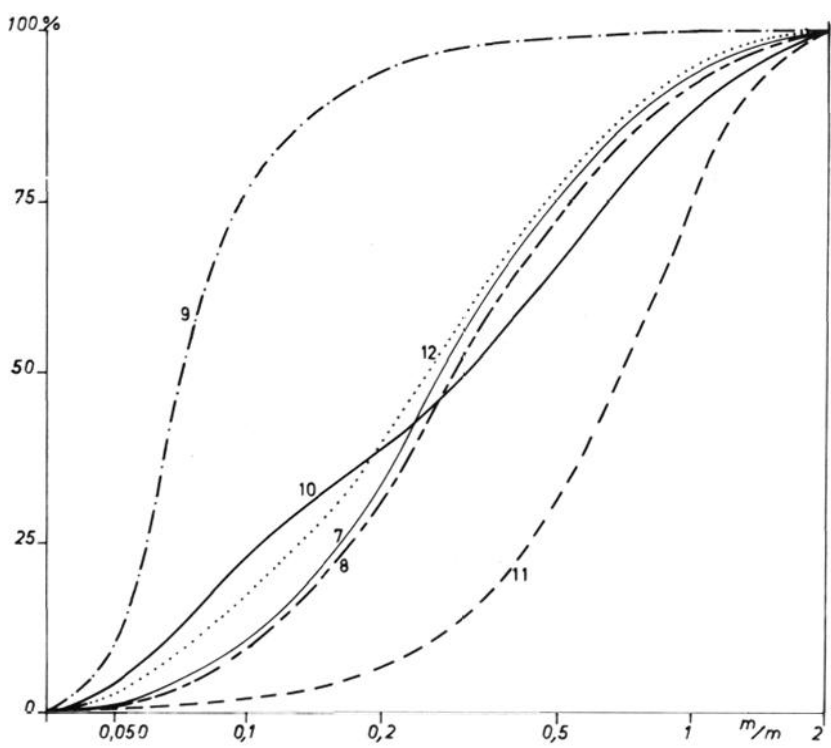

Fig. 3 : COURBES GRANULOMETRIQUE DES FRACTIONS SABLEUSES

Les chiffres renvoient aux subdivisions de la coupe.
Les courbes granulométriques des fractions sableuses (fig. 3) permettent de distinguer trois types de mise en place du matériel :

- la courbe du dépôt 9 est caractéristique d'un sable éolien très bien classé et très fin.

- les courbes des dépôts $7,8,10$ et 12 sont à peu près analogues. Elles représentent une mise en place de matériaux mélangés en régime aquatique. C'est l'essentiel du remblaiement de fond de vallée, qui provient de remaniements issus des versants. Mais alors que ces matériaux sont majoritaires à la base (tab. 1, dépôts 7 et 8), ils ne représentent plus que 20 à $30 \%$ du dépôt principal (10 et 12).

- la courbe du dépôt 11 est celle d'un sable fluviatile grossier bien classé.

La sédimentation de ce remblaiement de fond de vallée reste donc toujours plus ou moins sableuse, ce qui est logique dans le contexte considéré ; mais à des épisodes franchement détritiques, fluviatiles $(7,8,2,11)$ ou éoliens (9), s'opposent des passages à dépôts surtout limoneux $(10,12)$.

\section{Analyses qualitatives}

- Calcimétrie sur la fraction $<2 \mathrm{~mm}$ (sables, limons et argiles) : Tableau 2.

\begin{tabular}{|c|c|c|c|c|c|c|}
\hline Echantillons & 70,00 & 80,00 & 90,00 & 10,00 & 11,00 & 12,00 \\
\hline$\% \mathrm{Cac0}_{3}$ & 68,32 & 16,24 & 10,64 & 72,24 & 19,04 & 25,20 \\
\hline
\end{tabular}

Tab. 2 : POURCENTAGES DE $\mathrm{CaCO}_{3}$ DES FRACTIONS $<2 \mathrm{~mm}$

Les sables de la base (7) et la partie principale du dépôt limoneux (10) sont riches en calcaire.

- Diffractométrie $\mathrm{X}$ des argiles : Tableau 3.

Deux familles de dépôts argileux se distinguent, l'une à Ilite-kaolinite dominantes (8), de caractère très détritique, l'autre à Smectite-Attapulgite (tous les autres échantillons, 10 étant le plus riche en Smectite). Comme il s'agit de dépôt holocènes, ces argiles sont héritées. On peut cependant noter la bonne corrélation entre forts pourcentages en $\mathrm{CaCO}_{3}$ et présence des Smectites, classique dansles dépôts atlasiques (J. RISER, 1979).

- Analyse chimique sur sédiments <à $35 \mu \mathrm{m}$ (limons et argiles) : Tableau 4. 


\begin{tabular}{|c|c|c|c|c|c|}
\hline Echantillons & $\mathrm{K}$ & Att. & III. & $\mathrm{Sm}$. & Chlor. \\
\hline 12 & 7 & 26 & - & 67 & - \\
\hline 11 & 6 & 23 & - & 71 & - \\
\hline 10 & 3 & 17 & - & 80 & - \\
\hline 09 & 10 & - & 20 & 60 & 10 \\
\hline 08 & 41 & - & 52 & - & 07 \\
\hline 07 & 03 & 36 & - & 61 & - \\
\hline
\end{tabular}

Tab. 3 : ARGILES DES ECHANTILLONS

\begin{tabular}{|c|c|c|c|c|c|c|c|c|c|c|}
\hline Echant. & PF & $\mathrm{SiO} 2$ & $\mathrm{~A} \mid 203$ & $\mathrm{Fe} 203$ & Ti02 & $\mathrm{CaO}$ & $\mathrm{MgO}$ & $\mathrm{Na20}$ & K20 & Total \\
\hline 12 & 4,4 & 72,5 & 11,4 & 4,3 & 1,0 & 1,5 & 1,2 & 0,9 & 2,7 & 99,9 \\
\hline 11 & 3,8 & 72,6 & 11,9 & 3,8 & 1,1 & 1,3 & 1,1 & 1,1 & 2,5 & 99,2 \\
\hline 10 & 4,5 & 71,5 & 11.8 & 4,5 & 0,9 & 1,9 & 1,5 & 0,8 & 2,7 & 100,1 \\
\hline 09 & 19,0 & 44,5 & 7,4 & 3,0 & 1,1 & 21,6 & 1,0 & 0,6 & 1,2 & 99,4 \\
\hline 08 & 22,5 & 38,0 & 6,4 & 2,0 & 0,8 & 27,0 & 1,0 & 0,7 & 1,2 & 99,6 \\
\hline 07 & 06,0 & 69,1 & 11,5 & 4,3 & 1,0 & 3,7 & 1,2 & 0,8 & 2,5 & 100,1 \\
\hline
\end{tabular}

Tab. 4 : ANALYSES CHIMIQUES SUR LIMONS ET ARGILES

L'originalité des dépôts 8 et 9 se confirme par rapport au reste de la coupe, de composition assez homogène. Il y a donc eu une rupture marquée dans la sédimentation entre les dépôts 7 et 10.

Le dépôt principal du remblaiement de fond de vallon (10) est riche en calcaire dans sa fraction sableuse. Or celle-ci est composée de petits agrégats qui ont été observés en lames minces.

\section{II - ANALYSE TEXTURALE DU SÉDIMENT PRINCIPAL (Echantillon 10)}

\section{Observations en lames minces}

Les granules calcaires ont été préalablement ramollis (et non usés mécaniquement, ce qui entraîne des destructions irréversibles) avant d'être observés en lames minces. La structure granulaire et la porosité du matériel résultent de la juxtaposition de corps bactériens. Ces trames ne fournissent donc pas ici un usquelette» du type habituel des travertins sensu stricto, mais un matériau très comparable à celui des concrétions carbo. natées de fonds de vallées (J. P. ADOLPHE, 1981).

\section{In terprétation}

Le dépôt principal du remblaiement (Echantillons 10 et 12) est riche en calcaire dans sa fraction sableuse granulaire, ce qui indique une origine détritique, et non chimique de cette précipitation fixée par des 
micro-organismes bactériens. Le calcaire provient de la mobilisation des ciments de la molasse mio-pliocène ou de la karstification des dalles de croûte plio-quatemaires situées juste àl'amont. La précipitation s'est effectuée en régime aquatique à écoulements modérés et en régime calme, comme le suggère la présence de lits de Bivalves dulcicoles non dérangés. Ceci est en contraste flagrant avec les dépôts encadrants, nettement plus torrentiels, et annonçant les ruptures érosives antérieure et postérieure.

\section{L'âge du dépôt carbonaté principal :}

Une série de 6 datations $\mathrm{C}_{14}$ a été effectuée au Centre des Faibles Radio-activités de Gif/Yvette. 2 portent sur des charbons de bois et 4 sur des coquilles.

Les résul tats sont très cohérents dans les deux cas : les $60 \mathrm{~cm}$ médians du dépôt carbonaté, où s'intercalent de minces lentilles sableuses, se sédimentent entre $8160 \pm 80$ et $7480 \pm 350$ ans B.P. si l'on considère les charbons de bois et entre $11300 \pm 600$ et $9480 \pm 100$ ans B.P. selon les résultats foumis par les coquilles. On constate donc parfaitement sur cet exemple le kvieillissement" des âges donnés par les coquilles par rapport à ceux donnés par les charbons de bois.

Théoriquement, les coquilles terrestre sont impropres à la datation par le carbone 14 car le carbone que ces coquilles assimilent ne présente pas toujours une activité à l'équilibre avec celle du $\mathrm{CO}_{2}$ atmosphérique : on a montré que des escargots vivants pouvaient avoir des âges "apparents» de 800 à 1500 ans, suivant le milieu. Il y a presque toujours un "vieillissement" qui rend illusoire toute datation précise de mollusque terrestre. Si en archéologie c'est donc un nonsens de les dater, en stratigraphie cela donne néanmoins un repère chronologique. Enfin, ce "vieillissement» est constant quel que soit l'âge et est donc important surtout pour les âges assez récents.

Ceci montre donc qu'il semble préférable de s'en tenir aux résultats foumis par les charbons de bois, c'est-à-dire ici, en gros, entre 8000 et 7000 ans B.P. Par rapport à ce que l'on connaît par ailleurs dans la région, ces âges sont très satisfaisants : ils correspondent à ceux d'une récurrence humide qui a interrompu la grande aridification de l'Holocène inférieur (A. WEIROCK et al., 1985). Dans le cas précis du vallon de Makhfamane, cet épisode n'aboutit pas à la formation de travertins s.s., au développement ponctuel, mais à des dépôts carbonatés continus de fond de vallée, à concrétionnements sableux. C'est un type de dépôt qui marque une certaine régularisation du régime dans un milieu où la tendance générale est torrentielle.

\section{BIBLIOGRAPHIE}

ADOLPHE J P. (1981).- Exemples de contributions microorganiques dans les constructions carbonatées continentales. Actes du Colloque A:G.F. Formations carbonatées externes, tufs et travertins, Paris 9 mai 1981 , p. 15-30, 2 pl. photos.

DUTOUR A. (1985).- Formations alluviales et terrasses du Quaternaire moyen dans le Haouz de Marrakech (Maroc). Physiogéo, Paris, Actes du Colloque Quaternaire moyen A.G.F.et A.F.G.P.

RISER J. (1979).- L'évolution des minéraux argileux des niveaux quaternaires continentaux du Jbel Sarhro. Travaux Labo. Sc. de la Terre, Marseille-St-Jérôme, A, $\mathrm{n}^{\circ} 11,11 \mathrm{p}$.

WEISROCK A., DELIBRIAS G., ROGNON P., COUDE-GAUSSEN G. (1985).- Variations climatiques et morphogenèse au Maroc atlantique $\left(30-33^{\circ} \mathrm{N}\right)$ à la limite Pléistocène-Holocène. Bull. Soc. Géol. Fr. (8), t. I, n० 4 , p. 565-569. 